\title{
Elementary moves for higher dimensional knots
}

\author{
by
}

Dennis Roseman (Iowa City, IA)

\begin{abstract}
For smooth knottings of compact (not necessarily orientable) $n$-dimensional manifolds in $\mathbb{R}^{n+2}$ (or $\mathbb{S}^{n+2}$ ), we generalize the notion of knot moves to higher dimensions. This reproves and generalizes the Reidemeister moves of classical knot theory. We show that for any dimension there is a finite set of elementary isotopies, called moves, so that any isotopy is equivalent to a finite sequence of these moves.
\end{abstract}

1. Introduction. We consider (codimension two) smooth embeddings of a closed $n$-dimensional manifold $M^{n}$ into $\mathbb{R}^{n+2}$ or $\mathbb{S}^{n+2}$. Most of our results hold whether or not $M^{n}$ is orientable. Here $\mathbb{R}^{k}$ refers to the $k$-dimensional Euclidean space and $\mathbb{S}^{k}$ to the standard $k$-dimensional sphere, each with the standard differentiable structure. For any $k, \pi: \mathbb{R}^{k} \rightarrow \mathbb{R}^{k-1}$ denotes the standard projection onto the first $k-1$ coordinates.

Most commonly, one refers to a knot as an ambient isotopy class of an embedding; however, the word "knot" is also used to refer to an embedding which represents this class - or sometimes just the image of such an embedding. We adopt the following terminology:

DeFinition 1.1. A smooth codimension two embedding will be called a (smooth) knotting and the corresponding ambient isotopy class will be called a knot.

The smooth isotopy extension theorem (see, for example, [HR]) implies that a smooth isotopy gives rise to an ambient isotopy and we often use this without explicit mention.

Knotted circles were first studied by drawing projections of knots and studying changes called knot moves one could make in such projections which would yield the same knot. These ideas were (and still are) useful in generating examples and proving theorems (see [GR] for the early history of knot theory). In this paper we generalize these ideas to higher dimensions and discuss some examples.

2000 Mathematics Subject Classification: 57R40, 57R45, 57R52.

Key words and phrases: projections of knots, knot moves, Morse functions. 
We show that in all dimensions two projections of codimension two knottings correspond to the same knot if and only if one can change from one projection to the other by means of a finite collection of simple isotopies called moves.

Basic definitions from differentiable topology are found in $[\mathrm{HR}]$ and $[\mathrm{NB}]$.

Definitions and theorems on the projection of higher dimensional knots are to be found in [RS1]. In [RS2] we describe in detail the knot moves for two-manifolds in four-space, and consider the case of three-manifolds in five-space. These two papers together with the current one constituted the content of an unpublished monograph "Projections and Moves of Higher Dimensional Knots" referred to at the end of [RS1]. The author is very grateful to Józef Przytycki for his encouragement of these three publications.

2. Isotopies and knot moves. In Reidemeister's book [RD], it is shown that given a projection of a knot and an isotopy of it to another projection, the isotopy can be expressed using a finite sequence of moves $\Omega_{1}, \Omega_{2}, \Omega_{3}$, or their inverses (see Figure 1). As is traditional, Figure 1 shows only parts of the isotopy which change the double point structure of the projection in an essential way. We generalize this result to higher dimensional knots.
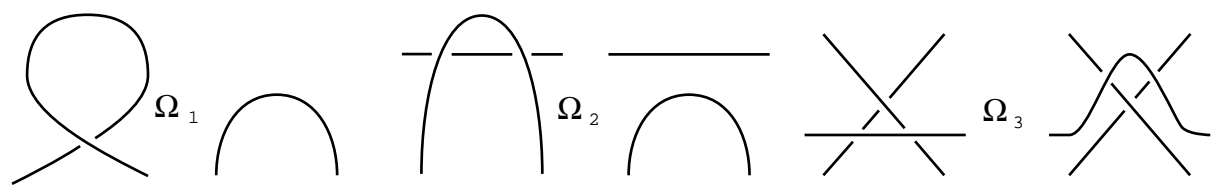

Fig. 1. The classical Reidemeister moves. Each of the above three equivalences is represented by only one possible choice of crossings.

The knot moves of Reidemeister are traditionally defined only by reference to a drawing rather than giving a formal definition of the concept of a knot move. Of course there is little need for a formal definition which has only three basic examples and whose nature can be easily and clearly expressed by very simple drawings with no need for other formalism. For higher dimensions however it is not as easy to communicate the nature of moves entirely by graphical means. In addition, the number of move types increases as one goes up in dimension. Thus we clearly need some more precise terminology.

Definition 2.1. Let $Y=\left\{Y_{i}\right\}_{i=1}^{k}$ be a disjoint collection of $n$-disks and let $F: Y \times I \rightarrow\left(B \times \mathbb{R}^{1}\right) \times I$ be a proper isotopy of $Y$ in $B \times \mathbb{R}^{1}$, where $B$ is an $(n+1)$-ball. Then we say $F$ is a local move. 
For example, the Reidemeister moves $\Omega_{1}, \Omega_{2}$, and $\Omega_{3}$ are local moves of (respectively) one, two and three proper 1-disks in $B \times \mathbb{R}^{1}$, where $B$ is a 2-disk.

An isotopy which, in the projection, moves subdisks of the embedded manifold within a ball will be called a move. To make this more precise we identify $\mathbb{R}^{n+2}$ as $\mathbb{R}^{n+1} \times \mathbb{R}^{1}$ and say that a $\operatorname{rod}$ is a subset $C \subseteq \mathbb{R}^{n+2}$ corresponding to $B \times \mathbb{R}^{1}$, where $B \subseteq \mathbb{R}^{n+1}$ is an $(n+1)$-ball. So $\bar{B}$ is the "cross-section of the rod".

Definition 2.2. An isotopy $F: M^{n} \times I \rightarrow \mathbb{R}^{n+2} \times I$ is a move with respect to $C$ if there is a rod $C$ such that $F^{-1}(C \times I)=Y \times I$, where $Y$ is a union of disjoint $n$-disks $\left\{Y_{i}\right\}_{i=1}^{k}$ in $M$ and $F \mid Y \times I$ is a local move.

Next, we discuss equivalence of isotopies. Let $\mathcal{E}\left(M^{n}, \mathbb{R}^{n+2}\right)$ denote the space of all smooth embeddings of $M^{n}$ in $\mathbb{R}^{n+2}$. A smooth isotopy of $M^{n}$ in $\mathbb{R}^{n+2}$ corresponds to a smooth path in $\mathcal{E}\left(M^{n}, \mathbb{R}^{n+2}\right)$.

Definition 2.3. Two isotopies are equivalent if the corresponding paths in $\mathcal{E}\left(M^{n}, \mathbb{R}^{n+2}\right)$ are smoothly path-homotopic.

We are interested in a finer equivalence - those isotopies whose projections look the same.

Definition 2.4. Let $f$ and $g$ be two knottings of $M$ in $\mathbb{R}^{n+2}$. We say $f$ and $g$ project equivalently if there is a homeomorphism $h: \mathbb{R}^{n+1} \rightarrow \mathbb{R}^{n+1}$ such that $\pi \circ g=h \circ \pi \circ f$.

Note that $h$ must send the crossing set of the knotting $f$ homeomorphically to the crossing set of the knotting $g$. (The notion of crossing set is defined in [RS1] and also in Definition 4.1.)

Definition 2.5. Two isotopies $f_{t}$ and $g_{t}$ project equivalently if there is an isotopy $h_{t}$ of $\mathbb{R}^{n+1}$ such that $\pi \circ g_{t}=h_{t} \circ \pi \circ f_{t}$ for all $t \in I$.

So, in particular, two isotopies project equivalently if they change the crossing sets in the same way. If two isotopies project equivalently, they are not necessarily equivalent, for example, we have $\pi \circ f_{0}=\pi \circ g_{0}$ and there is no requirement that $f_{0}$ and $g_{0}$ are isotopic. Figure 2 shows two isotopies of a pair of circles in $\mathbb{R}^{3}$ which are equivalent isotopies but do not project equivalently. Figure 3 shows three inequivalent isotopies which do project equivalently.

Definition 2.6. An isotopy projects without changes if there is an isotopy $h_{t}$ of $\mathbb{R}^{n+1}$ so that $\pi \circ f_{t}=h_{t} \circ \pi \circ f_{0}$ for all $t \in I$.

Note that in particular, the projection of this isotopy $h_{t}$ corresponds to an isotopy of $M^{*}=f_{0}(M)$ in $\mathbb{R}^{n+1}$. Thus neither of the isotopies in Figure 2 or 3 projects without changes. 


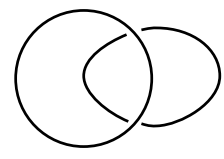

$f_{0}$

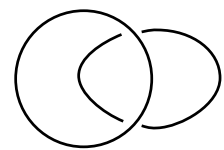

g。

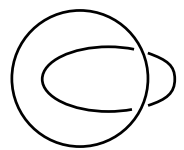

$f_{1 / 4}$

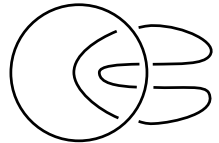

$g_{1 / 4}$

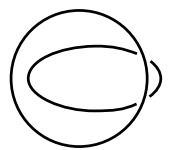

$\mathrm{f}_{1 / 2}$

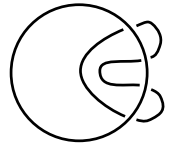

$\mathrm{g}_{1 / 2}$

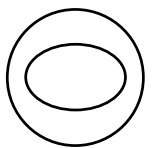

$f_{3 / 4}$

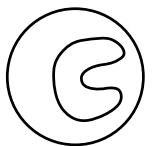

$g_{3 / 4}$

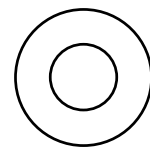

$f_{1}$

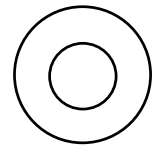

$g_{1}$

Fig. 2. $f$ and $g$ are equivalent isotopies, but in projection they are not the same. Note that for the projection of $f_{1 / 2}$ we have two crossing points while $g_{1 / 2}$ has four.
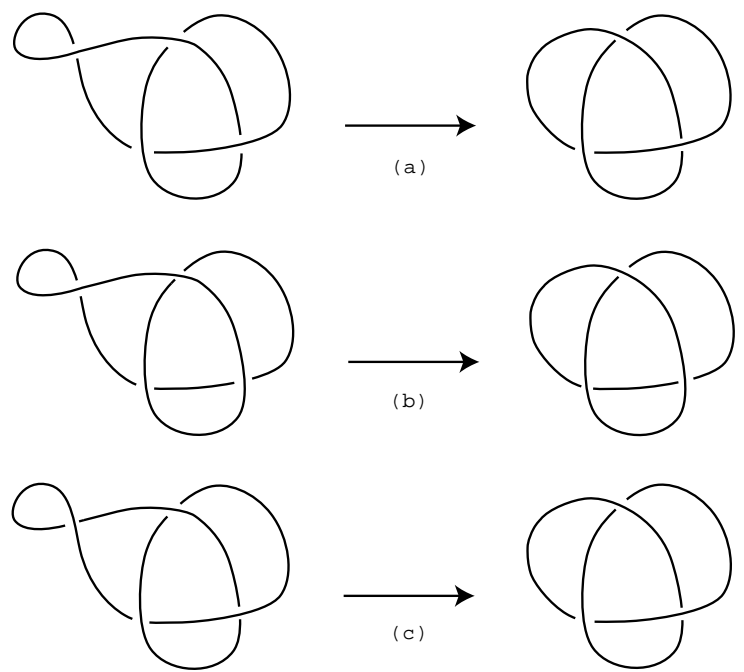

Fig. 3. Indicated are three isotopies whose projections are identical. Isotopies (a) and (c) are equivalent and project equivalently. Isotopy (b) is not equivalent to (a) or (c).

Finally, let $\mu$ be a move with respect to $C$, where $\mu: M^{n} \times I \rightarrow \mathbb{R}^{n+2} \times I$ and $Y$ and $B$ are as in Definitions 2.1 and 2.2.

Definition 2.7. Suppose $m: Y \times I \rightarrow\left(B \times \mathbb{R}^{1}\right) \times I$ is a given local move. Then we say $\mu$ projects equivalently with $m$ if $\mu \mid Y \times I$ projects equivalently with $m$ and $\mu \mid \overline{\left(M^{n}-Y\right)} \times I$ projects without changes.

REMARK 2.8. One effect of these definitions is "to ignore height relations for moves". That is, for example, the isotopies (a) and (c) of Figure 3 correspond to the same move. The advantage of this usage is to make smaller the number of moves. 
Proposition 2.9. For each dimension n, there is a finite set $\mathcal{M}^{n}$ of moves such that if $f_{0}$ and $f_{1}$ are any two codimension two knottings $M^{n} \subseteq$ $\mathbb{R}^{n+2}$ in general position with respect to projection, and $f_{t}$, where $t \in I$, is a smooth isotopy between $f_{0}$ and $f_{1}$, then $f$ is equivalent to an isotopy $g_{t}$ which is a concatenation of isotopies $g_{t}=\nu_{0} * \mu_{1} * \nu_{1} * \mu_{2} * \cdots * \mu_{k} * \nu_{k}$, where each $\mu_{i}$ is a move which projects equivalently with one of the elements of $\mathcal{M}^{n}$ and each $\nu_{i}$ is an isotopy which projects without changes.

These knot moves will be described in Section 6. We define:

Definition 2.10. The set $\mathcal{M}^{n}$ is called the set of standard local knot moves in dimension $n$.

REMARK 2.11. When $n=1$, this is just a form of the statement that "any isotopy can be obtained by a sequence of classical knot moves." In [RD] two additional moves $\Delta \pi_{1}$ and $\Delta \pi_{2}$ are needed. In our terminology, these moves project without changes and correspond to the $\nu_{j}$.

Our proof is somewhat technical. The reader is advised, on first reading, to consider Corollary 8.1 and Figure 4, and perhaps Corollary 8.2 for guides to the multidimensional situation.

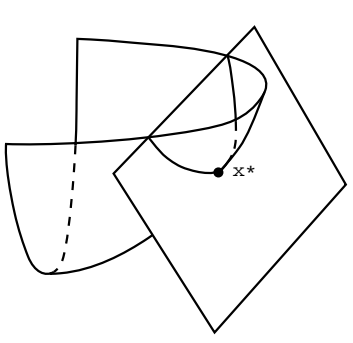

(a)

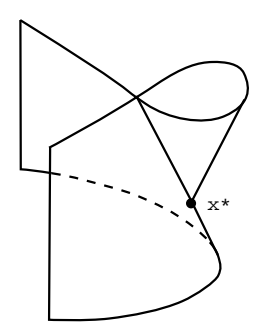

(b)

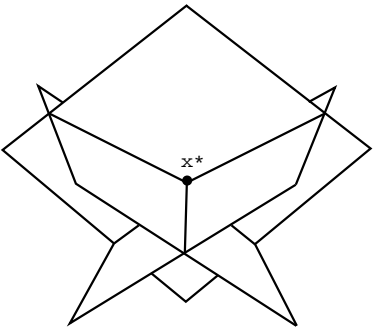

(c)

Fig. 4. Here $x^{*}$ denotes a critical point of the crossing set of the projection of the trace of an isotopy; (a) corresponds to $\Omega_{2}$, (b) to $\Omega_{1}$ and (c) to $\Omega_{3}$.

3. Outline of main theorem. Here is an outline of the proof of Proposition 2.9 .

1. View the isotopy $f_{t}$ as a map $F: M^{n} \times I \rightarrow \mathbb{R}^{n+2} \times I$. This is a codimension two knotting.

2. Put it in general position with respect to the projection $\pi^{\prime}: \mathbb{R}^{n+2} \times I$ $\rightarrow \mathbb{R}^{n+1} \times I$, where $\pi^{\prime}=\pi \times \operatorname{Id}_{I}$ - these concepts are defined in Section 4 .

3. Then we consider the corresponding double point set in $M \times I$ and arrange so that "the $I$ coordinate of this immersion is a Morse function," as is the $I$ coordinate on the branch set, and the $I$ coordinate of crossing sets of these. 
In order to have these knot moves occur in a sequence, rather than simultaneously, we alter our isotopy slightly so that each critical point has a distinct critical value; such an isotopy we refer to as "arranged for moves" - this notion is discussed in Section 5. The argument is a standard sort of argument about finding a "generic" map.

4. We classify the various possible types of critical point situations in Section 6 based on indices at these points.

5. The Morse Lemma says that a nondegenerate critical point after a change of coordinates can be modeled by a quadratic function. In Section 7 we use this to model neighborhoods of critical points to look like unions of graphs of quadratic coordinate functions. Thus we get geometric models near these critical points allowing a geometric picture of the knot moves.

Our technical concerns are basically two. Firstly, our definition of "generic" is lengthy, so we have several things to check. Secondly, we need to be careful that when we change our map $F$ we end up with an isotopy and not simply a nice cobordism.

4. General position. Let $f_{0}$ be a knotting of $M^{n}$ in $\mathbb{R}^{n+2}$ and suppose $f_{t}$ is an isotopy of $f_{0}$. Then we obtain an embedding $F: M \times I \rightarrow \mathbb{R}^{n+2} \times I$ defined by $F(x, t)=\left(f_{t}(x), t\right)$. We view $F$ as codimension two knotting by extending our consideration of knottings to include proper embeddings. For an isotopy $f_{t}$ we may assume without loss of generality that for some $\varepsilon>0$, $f_{t}=f_{0}$ if $0 \leq t \leq \varepsilon$ and $f_{t}=f_{1}$ if $1-\varepsilon \leq t \leq 1$. Thus, in particular, $F$ is a proper embedding. We will assume without loss of generality that all isotopies have this property.

We wish to speak of a general position with respect to a projection for an isotopy. For this, we recall some definitions from [RS1]. Examples found in [RS1] and [RS2] may be helpful in sorting out these definitions and notations.

Definition 4.1. Let $f: M^{n} \rightarrow \mathbb{R}^{n+2}$ be a smooth knotting. The projection of the knotting is the set $\pi \circ f(M)$, which we denote $M^{*}$. In general, if $A \subseteq M$, then $A^{*}$ denotes $\pi \circ f(A)$.

The crossing set of the knotting is the closure in $M^{*}$ of the set of all $x^{*} \in M^{*}$ such that $(f \circ \pi)^{-1}\left(x^{*}\right)$ contains two or more points. We denote this set by $D^{*}$.

The branch set of $f$, denoted by $B$, consists of all $x \in M$ such that $\pi \circ f$ is not an immersion at $x$.

The double point set of $M$ is $(\pi \circ f)^{-1}\left(D^{*}\right)$ and is denoted by $D$.

The double point set may contain points of higher order than two, as noted in Definition 4.6. 
Definition 4.2. Let $f: M^{n} \rightarrow \mathbb{R}^{n+2}$ be a smooth knotting with branch set $B$ and double point set $D$. We say $f$ is in general position with respect to the projection $\pi$ if

1. $B$ is a closed $(n-2)$-dimensional submanifold of $M^{n}$.

2. $D$ is the union of immersed closed $(n-1)$-dimensional submanifolds of $M^{n}$ with normal crossings. Denote the set of points of $D$ where normal crossings occur as $N$ and call this the self-crossing set of $D$.

3. $B$ is a submanifold of $D$ and for any $b_{0} \in B$ there is a small $(n-1)$ dimensional open subdisk $N$ with $b_{0} \in N, N \subseteq D$ such that $N-B$ has two components $N_{0}$ and $N_{1}$, each of which is an $(n-1)$-disk which is embedded by the restriction of $\pi \circ f$ but with $N_{0}^{*}=N_{1}^{*}$.

4. $B$ meets $N$ transversely.

5. $(\pi \circ f) \mid B$ is an immersion of $B$ with normal crossings.

6. The crossing set of $B^{*}$ is transverse to the crossing set of $M^{*}$.

The simplest example for $n=2$ of a projection of a branch point is shown in Figure 4(b). Condition 3 above says that at a neighborhood of a branch point the projection of a neighborhood of a branch point looks like the cartesian product of that model and $\mathbb{R}^{n-2}$.

Note that the projection of the double point set is the crossing set. For the knottings in general position with respect to projection we have $B \subseteq D$. We also adopt similar definitions and notations for the projection $\pi^{\prime}$.

Also in [RS1] we give examples and prove the following general position result.

Proposition 4.3 (General position for knottings). Given a knotting $F: M^{n} \rightarrow \mathbb{R}^{n+2}$ we may isotope $F$ to a map which is in general position with respect to projection.

We can now define what it means for an isotopy $F$ to be in general position with respect to the projection $\pi^{\prime}$. It is just the previous definition for general position of a codimension two knotting except that $B$ and $D$ may have nonempty boundary. Also the manifolds $B, D$ as well as the double point set of $B$ and crossing set of $D$ are proper submanifolds. In addition, at points $b_{0} \in \partial B$, we find a neighborhood $N$ of $b_{0}$ homeomorphic to a half-open disk (as in condition 3 of the definition of general position with respect to projection).

Proposition 4.4. Any isotopy $F: M^{n} \times I \rightarrow \mathbb{R}^{n+2} \times I$ is equivalent to an isotopy $G$ which is in general position with respect to projection. Furthermore, if $F \mid M^{n} \times\{0\}$ and $F \mid M^{n} \times\{1\}$ are in general position with respect to projection, we may find such a $G$ which is equivalent to $F$ and with $G\left|M^{n} \times\{0\}=F\right| M^{n} \times\{0\}$ and $G\left|M^{n} \times\{1\}=F\right| M^{n} \times\{1\}$. 
Proof. The proof proceeds much like that for Theorem 3.1 of [RS1]. We find a knotting $F^{\prime}$ of $M^{n} \times I$ close to $F$ which is in general position with respect to the projection $\pi^{\prime}$. The fact that $M^{n} \times I, \mathbb{R}^{n+2} \times I$ and $\mathbb{R}^{n+1} \times I$ are manifolds with boundary is not a problem. However we must be careful and make sure that $F^{\prime}$ is level preserving and thus an isotopy. This is done by the following proposition which shows that a map of $M^{n} \times I$ which is close to an isotopy is an isotopy.

Proposition 4.5. Let $F: M^{n} \times I \rightarrow \mathbb{R}^{n+2} \times I$ be an isotopy. Then there is a number $\varepsilon>0$ such that if $G: M^{n} \times I \rightarrow \mathbb{R}^{n+2} \times I$ is a proper embedding with $\delta(F, G)<\varepsilon$ (where $\delta$ denotes the $\mathcal{C}^{\prime}$ metric), then $G$ is an isotopy. If $F\left|M^{n} \times\{0\}=G\right| M^{n} \times\{0\}$ and $F\left|M^{n} \times\{1\}=G\right| M^{n} \times\{1\}$, then $G$ is equivalent to $F$.

Proof. Let $p: \mathbb{R}^{n+2} \times I \rightarrow I$ be projection. Consider $p \circ F$. This is regular at each point of $M^{n} \times I$ since the partial derivative in the $I$ direction is never zero. So there is an $\varepsilon>0$ such that for $\delta(F, G)<\varepsilon, G$ is also regular at each point of $M^{n} \times I$. Thus each set $(G \circ p)^{-1}(t)$ is a manifold diffeomorphic to $M^{n}$, giving a product structure to $M^{n} \times I$ (for example, think of $G \circ p$ as a Morse function with no critical points and refer to Milnor $[\mathrm{ML}])$.

We now suppose that $F \mid M^{n} \times\{0\}$ and $F \mid M^{n} \times\{1\}$ were in general position with respect to projection and consider the second assertion of the lemma. Since we may assume the isotopy is constant near 0 and near 1 , we may assume the $G$ we obtain above agrees with $F$ near 0 and near 1 . A relative version of Lemma 3.2 of [RS1] shows that $G$ is path homotopic to $F$ in the space of embeddings of triples $\left(M^{n} \times I, M^{n} \times\{0\}, M^{n} \times\{1\}\right) \rightarrow$ $\left(\mathbb{R}^{n+2} \times I, \mathbb{R}^{n+2} \times\{0\}, \mathbb{R}^{n+2} \times\{1\}\right)$ by a path $\alpha(t)$. Since we may make this path as small as we wish, we may make sure that for each $t$, the embedding $\alpha(t)$ satisfies the conditions of Proposition 4.5. Thus $\alpha_{t}$ is a one-parameter family of isotopies from $F$ to $G$.

To have an isotopy $F$ in general position with respect to projection is not sufficient for our purposes. We also want to make requirements on the double point set in the $I$ direction in $M^{n} \times I$. It is easiest to describe this using the crossing set.

We need some more terminology for certain sets (see [RS1] for more information). Roughly speaking, " $D$ and $D^{*}$ are unions of $(n-1)$-dimensional manifolds and $\pi \circ \phi \mid D$ is basically a two-to-one map." The following terminology makes this more precise.

Definition 4.6. Let $F: M^{n} \rightarrow \mathbb{R}^{n+2}$ be a knotting in general position with respect to projection $\pi$. Define $T=\left\{x \in M^{n} \mid(\pi \circ F)^{-1}\left(x^{*}\right)\right.$ contains three points or more $\}$. We call $T$ the triple point set. Let $P=D-(B \cup T)$. 
We call $P$ the set of pure double points $-P$ is the set such that $\pi \circ F$ is exactly two-to-one.

It follows from the definition of general position that $P$ is an open dense $(n-1)$-submanifold of $D$. As usual, we let $P^{*}=(\pi \circ F)(P)$. Then the statement, " $D^{*}$ is the union of immersed $(n-1)$-dimensional manifolds," can be expressed as follows. There is a compact $(n-1)$-dimensional manifold $\Gamma$ and an immersion $\gamma: \Gamma \rightarrow \mathbb{R}^{n+1}$ such that $\gamma(\Gamma)=D^{*}$ and $\gamma \mid \gamma^{-1}\left(P^{*}\right)$ is one-to-one. Let $\Gamma_{i}$ be the components of $\Gamma$, let $\gamma_{i}=\gamma \mid \Gamma_{i}$ and let $D_{i}^{*}=$ $\gamma\left(\Gamma_{i}\right)$. Similarly let $\Delta$ be an $(n-1)$-dimensional manifold with $\delta: \Delta \rightarrow M$ an immersion such that $\delta(\Delta)=D$ and $\delta \mid \delta^{-1}(P)$ is one-to-one. Let $D_{i}=$ $(\pi \circ F)^{-1}\left(D_{i}^{*}\right)$ and let $\Delta_{i}$ denote $\delta^{-1}\left(D_{i}\right)$. There is a unique map $\alpha: \Delta \rightarrow \Gamma$ such that the following diagram commutes:

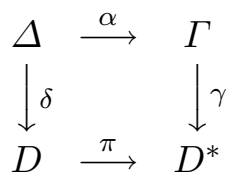

Also note that $\alpha \mid \Delta-\delta^{-1}(B)$ is a two-to-one map onto $\Gamma-\gamma^{-1}\left(B^{*}\right)$.

Some examples such as Figure 7 of [RS1] might clarify the above ideas and notations.

Definition 4.7. Let $h: \mathbb{R}^{n+1} \rightarrow \mathbb{R}^{1}$ be projection on the last coordinate; we refer to $h$ as the height function. Suppose $z \in \Gamma$ with $\alpha^{-1}(z)$ consisting of two points, $\widetilde{x}$ and $\widetilde{x^{\prime}}$. Then we call these points points paired by projection.

Tracing through our definitions we can verify that $x=\delta(\widetilde{x})$ and $x^{\prime}=$ $\delta\left(\widetilde{x^{\prime}}\right)$ are distinct points in $M$. Since $F$ is a knotting, we must have $h(F(x)) \neq$ $h\left(F\left(x^{\prime}\right)\right)$.

Definition 4.8. If $h(F(x))>h\left(F\left(x^{\prime}\right)\right)$ we say that $\widetilde{x}$ is an over point. Otherwise $\widetilde{x}$ is an under point. Similarly we also say that $x$ is an over point and $x^{\prime}$ is an under point.

In [RS1] the following was shown:

Proposition 4.9. If $\partial \Gamma_{i}=\emptyset$, then $\Delta_{i}$ has two components $\Delta_{i}^{+}$and $\Delta_{i}^{-}$, where $\Delta_{i}^{+}$is a set of over points and $\Delta_{i}^{-}$is a set of corresponding under points, with $\pi \circ F \circ \delta\left(\Delta_{i}^{+}\right)=\pi \circ F \circ \delta\left(\Delta_{i}^{-}\right)=D_{i}^{*}$.

If $\partial \Gamma_{i} \neq \emptyset$ then $\Delta_{i}-\delta^{-1}(B)$ has two components $\Delta_{i}^{+}$and $\Delta_{i}^{-}$, where $\Delta_{i}^{+}$is a set of over points and $\Delta_{i}^{-}$is a set of corresponding under points such that $\pi \circ F \circ \delta\left(\Delta_{i}^{+}\right)=\pi \circ F \circ \delta\left(\Delta_{i}^{-}\right)=D_{i}^{*}$.

In either case, we let $D_{i}^{+}=\delta\left(\Delta_{i}^{+}\right)$and $D_{i}^{-}=\delta\left(\Delta_{i}^{-}\right)$. 
5. Arranging for moves. We may extend the definitions of $D_{i}, D_{i}^{+}$, $D_{i}^{-}, B_{i}, \Gamma_{i}, \delta$, and $\gamma$ to proper knottings. In particular we have in mind the trace of an isotopy $M \times I \rightarrow \mathbb{R}^{n+2} \times I$ which is in general position with respect to projection $p: \mathbb{R}^{n+2} \times I \rightarrow I$.

Definition 5.1. Let $q$ be a proper immersion of a manifold $Q$ in $\mathbb{R}^{n+1} \times I$. We say that $q(Q)$ is immersed Morse style if $p \circ q$ is a Morse function.

Suppose $F$ is an isotopy in general position with respect to projection. We define $Q^{(0)}$ to be the crossing set of $B^{*}, Q^{(1)}=B^{*}$. For $k>1, Q^{(k)}$ is the closure of the subset of $D^{*}$ such that $\pi^{\prime} \circ F$ is at least $k$-to-one. In particular, $Q^{(2)}$ consists of $D$.

Also, $Q^{(3)}$ corresponds to:

- the triple points of $M^{*}$;

- the intersection of $B^{*}-\left(\operatorname{crossing}\right.$ set of $\left.B^{*}\right)$ and the crossing set of $D^{*}$;

- the intersection of the crossing set of $B^{*}$ and $D-\left(\right.$ crossing set of $\left.D^{*}\right)$.

In the cases where $n \leq 3, B^{*}$ is an immersed submanifold having normal crossings. This implies that $B^{*}$ has empty self-crossing set. When $n=3$, $B^{*}$ will be a circle or union of circles in a 3-manifold with no self-intersection. However self-crossings of $B^{*}$ are possible if $n \leq 4$. For example, suppose $n=4$; we are considering the projection into $\mathbb{R}^{5}$ of a 4 -manifold $M^{4}$ knotted in $\mathbb{R}^{6}$. The crossing set $D^{*}$ will be a union of immersed 3-manifolds. The self-intersection set $T^{*}$ of the crossing set (generically triple points of the projection) will be 2 -dimensional. If we have $B^{*} \neq \emptyset$ then $B^{*}$ will correspond to the boundaries of components of these crossing set manifolds. So $B^{*}$ will be an immersed 2-manifold in a 4-manifold and might have generic points of self-intersection. In this case $B^{*}$ might intersect $D^{*}$ transversely in a 1-dimensional set and might intersect $T^{*}$ in a finite number of points.

In any case, each $Q^{(i)}$ is the union of a finite number of immersed manifolds $Q_{j}^{(i)}$, where for a fixed $i$ these manifolds may be of different dimensions. For $k \geq 2$ let $Q_{\#}^{(k)}=Q^{(k)}-Q^{(k-1)}$. We note that $Q_{\#}^{(k)}$ is open and dense in $Q^{(k)}$.

In the definition below we make the convention that any real-valued function defined on a zero-dimensional manifold is a Morse function; furthermore, every such point will be considered to be a critical point of index 0 . In addition, if $f$ is a real-valued function defined on $Q$ with $\partial Q \neq 0$, we say $f$ is Morse if $f \mid Q-\partial Q$ and $f \mid \partial Q$ are Morse and these have no common critical points (that is, if $x \in \partial Q$ is a critical point of $f \mid \partial Q$ then the derivative of $f$ in the direction normal to $\partial Q$ is nonzero). 
Definition 5.2. We say that $F$ is arranged for moves if

1. $F$ is in general position with respect to projection.

2. For all $i$ and $j, Q_{j}^{(i)}$ is immersed Morse style.

3. The critical values of all the Morse functions involved with condition 2 above are all distinct with none equal to zero or one.

4. For $i \geq 2$, all critical points of $Q^{(i)}$ lie in $Q_{\#}^{(i)}$.

Definition 5.3. We call the critical points of the Morse functions involved in condition 2 of Definition 5 elementary singularities; the index of the elementary singularity is the index of the critical point.

Being arranged for moves is not at all special - in fact it is generic:

Proposition 5.4. Given any isotopy $F: M^{n} \times I \rightarrow \mathbb{R}^{n+2} \times I$ in general position with respect to projection, we may find an equivalent isotopy $G$ which is arranged for moves.

Proof. It is convenient to take advantage of the natural embedding $\nu: \mathbb{R}^{n+2} \times I \rightarrow \mathbb{R}^{n+3}$. Our proof will be based on the proof of existence of Morse functions as found in [NB], namely, if $P$ is a submanifold of $\mathbb{R}^{m}$ and $f: P \rightarrow \mathbb{R}^{1}$ a smooth function, then $f+\ell$ is a Morse function on $P$ for most linear functions $\ell: \mathbb{R}^{m} \rightarrow \mathbb{R}^{1}$. Our technical problems here are that the manifolds $Q^{(i)}$ have possibly nonempty boundary, and they are immersed, not necessarily embedded.

Let $Q=\bigcup_{i} Q^{(i)}, q^{*} \in Q \cap\left(\mathbb{R}^{n+1} \times(0,1)\right)$, and let $N$ be a small open ball in $\mathbb{R}^{n+2} \times I$ containing $q^{*}$. Then $f\left(M^{n} \times I\right) \cap N$ looks like the union of $k$ sets $\left\{W_{i}\right\}_{i=1}^{k}$ so that each $W_{i}$ is an $(n+1)$-dimensional disk or a halfdisk, with the collection in general position. We wish to describe the set on which singularities are allowed to occur-this excludes points at the halfdisks not on the boundary and includes all possible intersections of disks and boundaries of half-disks. We next define $\left\{W_{i}^{\prime}\right\}_{i=1}^{k^{\prime}}$ to be the union of $\left\{W_{i}\right\}_{i=1}^{k}$ together with all boundary $n$-disks of those $W_{i}$ (if any) which are half-disks. Let $\left\{Y_{j}\right\}_{j=1}^{s}$ be the union of $\left\{W_{i}^{\prime}\right\}_{i=1}^{k}$ and the collection of sets obtained by intersecting two or more of the $\left\{W_{i}^{\prime}\right\}_{i=1}^{k}$.

Now consider some $Y_{j_{0}}$ and suppose it is an embedded disk of dimension $r$. Viewing $Y_{j_{0}} \subseteq \mathbb{R}^{n+3}$ we may assume this embedding is given by $\left(\phi_{1}(x), \ldots, \phi_{n+3}(x)\right)$, where $x \in D^{r}$.

The map $p: \mathbb{R}^{n+2} \times I \rightarrow I$ now corresponds to $\phi_{n+3}(x)$. As in [NB], for almost all $\vec{a} \in \mathbb{R}^{n+3}, \vec{a}=\left(a_{1}, \ldots, a_{n+3}\right)$, the function

$$
\phi_{n+3}^{\left(j_{0}\right)}(x)=\phi_{n+3}(x)+a_{1} \phi_{1}(x)+a_{2} \phi_{2}(x)+\cdots+a_{n+3} \phi_{n+3}(x)
$$

is Morse. It is easy to see that for almost all $\vec{a} \in \mathbb{R}^{n+3}, \phi_{n+3}^{\left(j_{0}\right)}(x)$ is Morse with all critical values distinct. Furthermore, since $Q_{\#}^{(i)}$ is open and dense 
in $Q^{(i)}$ we may assume that these critical points, if they are critical points of $Q^{(i)}$, correspond to points of $Q_{\#}^{(i)}$. Since there are only finitely many $Y_{j}$, it follows that for almost all $\vec{a} \in \mathbb{R}^{n+3}$ we have all $\phi_{n+3}^{(j)}(x)$ Morse functions with all critical points having distinct critical values.

Recall our assumption that the isotopy $F$ is constant within some $\varepsilon$ of 0 or 1 ; we wish our new isotopy $G$ to have this property also. Let $U=M^{n} \times(\varepsilon / 2,1-\varepsilon / 2)$ and $U^{\prime}=M^{n} \times([0, \varepsilon) \cup(1-\varepsilon, 1])$. Consider $\nu \circ F: M^{n} \times I \rightarrow \mathbb{R}^{n+3}$; suppose $\nu \circ F(x)=\left(f_{1}(x), \ldots, f_{n+3}(x)\right)$, here $f_{n+3}(x)$ corresponds to the projection $p$. Suppose $\vec{a} \in \mathbb{R}^{n+3}$, and let $f_{n+3}^{\prime}(x)=$ $f_{n+3}(x)+a_{1} f_{1}(x)+\cdots+a_{n+3} \phi_{n+3}(x)$. Then by using a finite cover of $N \cup F\left(M^{n} \times I\right)$ we can see that for almost all $\vec{a} \in \mathbb{R}^{n+3}, f_{n+3}^{\prime}(x)$ will restrict to a Morse function on $Q$ with all critical values distinct, and with critical points of $Q^{(i)}$ belonging to $Q_{\#}^{(i)}$.

To obtain our isotopy $G$ we proceed as follows. Choose an $\vec{a} \in \mathbb{R}^{n+3}$ close to $(0,0, \ldots, 0,1)$, thus $f_{n+3}^{\prime}(x)$ is close to $f_{n+3}(x)$, and the function $F^{\prime}: M^{n} \times I \rightarrow \mathbb{R}^{n+3}$ defined by $F^{\prime}(x)=\left(f_{1}(x), \ldots, f_{n+2}(x), f_{n+3}^{\prime}(x)\right)$ is close to $F$. So $F$ is an embedding in general position with respect to projection. Furthermore, we should choose $\vec{a}$ small enough so that $F^{\prime}(\bar{U}) \subseteq$ $\nu\left(\mathbb{R}^{n+2} \times(0,1)\right)$. In the usual way we can define $G$ by patching together $F \mid \overline{\bar{U}}$ and $F \mid U^{\prime}$ by a smooth partition of unity subordinate to the cover $\left\{U, U^{\prime}\right\}$. By Proposition 4.4, $G$ is an isotopy equivalent to $F$. By using reasonable care in our partition of unity, we introduce no additional critical points on $Q$ and thus we have the desired isotopy.

REMARK 5.5. We defined our notion of arranged for moves in terms of the crossing set $D^{*}$ of the map $F: M^{n} \times I \rightarrow \mathbb{R}^{n+2} \times I$. Let us consider the corresponding double point set $D$. Of course $p \circ(F \mid D)$ is a Morse function on $D$. In $D-B$ it is clear that the singularities of the Morse function are just like those on $D^{*}$ except there will be twice as many. However on points of $B$, since there is a "fold" along $B$, we note that if $b_{*}$ is a critical point of $B^{*}$ then it is not (by our definition) a critical point of $D^{*}$, but that $b$ must be a critical point of $D$ (since as we can see from Morin's [MR] local coordinates about $b$, a small curve in $D$ transverse to $B$ at $b$ must either have a local minimum or a local maximum at $b)$.

6. Listing the knot moves. We are now ready to describe a standard set $\mathcal{M}^{n}$ of moves for each dimension. Fix a dimension $n$ and suppose we are given an isotopy $F: M^{n} \times I \rightarrow \mathbb{R}^{n+2} \times I$ which is arranged for moves. This gives a sequence of elementary singularities. Each singularity will correspond to a standard local knot move in our collection $\mathcal{M}^{n}$. 
In the notation which follows, we consider three general types of points:

1. branch type: critical points of $B^{*}$ and self-crossing points of $B^{*}$ for which we use the letter $b$;

2. crossing type: critical points of $D^{*}$ and the crossing set of $D^{*}$ which do not belong to $B^{*}$ for which we use the letter $c$;

3. mixed type: critical points which are in the crossing set of $D^{*}$ and are in $B^{*}$, a "mixed" type for which we use the letter $m$.

The first collection of branch type points is denoted $\{S(b, k, p, q)\}$. If $x^{*} \in D^{*}$ is such a singular point, where $D^{*}$ is the crossing set of $F: M^{n} \times I \rightarrow$ $\mathbb{R}^{n+1} \times I$, let $k$ denote the number of points of $F^{-1}\left(x^{*}\right)$. In our case the branch point set of $F$, since it is of codimension 2 in $\mathbb{R}^{n+1} \times I$, will be of dimension $n-1$. If in projection this branch set intersects itself generically, the self-intersection set will have dimension $n-4$. It follows that $1 \leq k$ $\leq n-2$. The integer $p$ is the index of the singularity. The integer $q$ has range $0 \leq q \leq k$ and might be called transverse index of this critical point. This is defined as follows. If $x \in B$ consider a curve $\delta$ in $D$ transverse to $B$ (recall that $B$ has codimension one in $D$ ) so that $\delta^{*}$ is, except for the point $x$, the two-to-one image of $\delta$. In the $I$ direction, the image of this curve has a local maximum or a local minimum at $b^{*}$. Now suppose $b^{*}$ is a $k$-fold point of $B^{*}$; then we have $k$ such curves to consider. The number $q$ is the number of those curves for which we have a local maximum. Of course, it follows that $k-q$ of the curves have a local minimum.

Consider $S(b, 1,0,1)$, for example. The symbols inside the parentheses inform us that we have an elementary singularity $x^{*}$ with regard to the $I$ coordinate:

1. $x^{*} \in B^{*}$.

2. $x^{*}$ is not in the crossing set of $B^{*}$.

3. $B$ has a local minimum at $x$.

4. For a curve $\delta$ at $x$ in $D$ transverse to $B$, we have a local maximum. Similarly for a singularity $x^{*}$ of type $S(b, 2,1,1)$ :

1. $x^{*} \in B^{*}$.

2. $x^{*}$ is a crossing point (but not a triple point) of $B^{*}$.

3. $B$ has a singularity of index 1 at $x$.

4. Two disks of $B^{*}$ intersect transversely at $x^{*}$; one disk has an $\operatorname{arc} \delta$ in $D$ transverse to $B$ at $x$ with a local minimum in the $I$ direction, and for the other disk, a similar arc $\delta^{\prime}$ has a local maximum.

The next collection of crossing type singularities is denoted by $\{S(c, k, p)\}$. If $x^{*}$ is such a singularity, $k$ denotes the cardinality of $F^{-1}(x)$. Thus $k$ is an integer with $2 \leq k \leq n+2$. Furthermore, on the set of points where $F$ is $k$-to-one, $x^{*}$ is a critical point in the $I$ direction, of index $p$. 
Finally, $S(m,(i, j), p, q)$ denotes the mixed singularities. Such a singularity $x^{*}$ has $F^{-1}(x)$ consisting of $i+j$ points, exactly $i$ of which are in $B$. Again $p$ is the index of the singularity and $q$ is an integer with $0 \leq q \leq i$ which is the number of local maxima we get by looking at those $i$ arcs transverse to $B$ at the points of $F^{-1}\left(x^{*}\right) \cap B$.

We next discuss, in detail, the singularity types $S(c, 2, p)$ and $S(b, 1, p, q)$.

A singularity of type $S(c, 2, p)$ corresponds to the following situation: we have locally two $(n+1)$-disks intersecting transversely in an $n$-disk and on that $n$-disk we have a single Morse singularity of index $p$. In this case, our move yields isotopies of two disks $Y_{1}$ and $Y_{2}$, that is, maps $Y_{i} \times I \rightarrow$ $\left(B_{0} \times \mathbb{R}^{1}\right) \times I$, where $B_{0}$ is an $(n+1)$-ball.

However, it is more convenient to first define maps $\mathbb{R}^{n} \times \mathbb{R}^{1} \rightarrow \mathbb{R}^{n+2} \times \mathbb{R}^{1}$ and obtain our isotopies by restriction of domain and reparameterization of the last coordinate. Let $\mu_{p}^{n}$ be the standard function in $n$-variables giving a Morse function with one critical point of index $p$ and critical value zero at the origin; specifically

$$
\mu_{p}^{n}\left(t_{1}, \ldots, t_{n}\right)=\sum_{i=1}^{p}-t_{i}^{2}+\sum_{i=p+1}^{n} t_{i}^{2} .
$$

Next, define two maps $f_{1}, f_{2}: \mathbb{R}^{n} \times \mathbb{R}^{1} \rightarrow \mathbb{R}^{n+2} \times \mathbb{R}^{1}$ by

$$
\begin{aligned}
& f_{1}\left(\left(t_{1}, \ldots, t_{n}\right), s\right)=\left(\left(t_{1}, \ldots, t_{n}, s, 1\right), \mu_{p}^{n}\left(t_{1}, \ldots, t_{n}\right)-s\right), \\
& f_{2}\left(\left(t_{1}, \ldots, t_{n}\right), s\right)=\left(\left(t_{1}, \ldots, t_{n}, s, 0\right), \mu_{p}^{n}\left(t_{1}, \ldots, t_{n}\right)-s\right) .
\end{aligned}
$$

Clearly each of $f_{1}$ and $f_{2}$ is an embedding and the images are disjoint since they differ in the last coordinate of $\mathbb{R}^{n+2}$. If $P: \mathbb{R}^{n} \times \mathbb{R}^{1} \rightarrow \mathbb{R}^{n+2} \times \mathbb{R}^{1}$ denotes the map obtained by projection along the last coordinate of $\mathbb{R}^{n+2}$, then the images of $P \circ f_{1}$ and $P \circ f_{2}$ intersect transversely in a set homeomorphic to $\mathbb{R}^{n}$. In fact, the intersection is the image of $P \circ f_{i}\left(\mathbb{R}^{n} \times\{0\}\right)$, where $i=1$ or 2 . Furthermore, if $h: \mathbb{R}^{n+1} \times \mathbb{R}^{1} \rightarrow \mathbb{R}^{1}$ is projection onto the last factor, then

$$
h \circ P \circ f_{i}\left(t_{1}, \ldots, t_{n}, 0\right)=\mu_{p}^{n}\left(t_{1}, \ldots, t_{n}\right) .
$$

The maps $f_{i}: \mathbb{R}^{n} \times \mathbb{R}^{1} \rightarrow \mathbb{R}^{n+2} \times \mathbb{R}^{1}$ are not level preserving (that is, do not preserve the $\mathbb{R}^{1}$ coordinate), however, if $s \in \mathbb{R}^{1}$ then $f_{i}^{-1}(s)$ is diffeomorphic to $\mathbb{R}^{n}$. For example, $f_{1}^{-1}(0)$ is just the graph of $\mu_{p}^{n}$. In general $f_{i}^{-1}(s)$ is the graph of $\mu_{p}^{n}+c$ for some constant $c$. Thus if $C$ is an $(n+1)$-ball containing the origin in $\mathbb{R}^{n+1}$ and $D^{1}=[-1,1]$ then $f_{i}^{-1}\left(\left(C \times \mathbb{R}^{1}\right) \times D^{1}\right)$ is homeomorphic to $Y_{i} \times D^{1}$, where $Y_{i}$ is an $(n+1)$-ball.

If we reparameterize $D^{1}$ to $I$ and denote disjoint union by $\cup_{0}$ then the map

$$
\phi:\left(Y_{1} \times I\right) \underset{\circ}{\cup}\left(Y_{2} \times I\right) \rightarrow \mathbb{R}^{n+2} \times I
$$

such that $\phi \mid Y_{i} \times I=f_{i}$ is our standard local knot move corresponding to the singularity $S(c, 2, p)$. 
Next we consider a singularity of type $S(b, 1, p, q)$; locally this singularity corresponds to an $(n+1)$-disk with branch set which is an $(n-1)$-disk, and on that $(n-1)$-disk we have a single Morse singularity of index $p$. In this case, our standard local move is an isotopy of a single disk. We proceed as with the previous case.

In this case, we define $f: \mathbb{R}^{n} \times \mathbb{R}^{1} \rightarrow \mathbb{R}^{n+2} \times \mathbb{R}^{1}$ by

$f\left(\left(t_{1}, \ldots, t_{n-1}, x\right), s\right)=\left(\left(t_{1}, \ldots, t_{n-1}, t_{1} x, x^{2}, x\right), \mu_{p}^{n-2}\left(t_{2}, \ldots, t_{n-1}\right)+\varepsilon x^{2}+s\right)$.

Here $\varepsilon=1$ if $q=0$, and $\varepsilon=-1$ if $q=1$. It is easy to check that $f$ is an embedding and that $P \circ f$ is an image of an $(n+1)$-disk with an $(n-1)$ subdisk of canonical branch points. This branch set corresponds to the points $\left(0, t_{2}, \ldots, t_{n}, 0, s\right)$ and the double point set of this map is the set of points $\left(0, t_{2}, \ldots, t_{n-1}, x, s\right)$. As before, we note that $f^{-1}(s)$ is diffeomorphic to $\mathbb{R}^{n}$ for $s \in \mathbb{R}^{1}$. For example, $f^{-1}(0)$ is the graph of $\mu_{p}^{n-2}\left(t_{2}, \ldots, t_{n-1}\right)+\varepsilon x^{2}=s$, where we view $s$ as a function of $\left(t_{1}, \ldots, t_{n-1}, x\right)$. We then proceed as in the previous case to obtain a local move $\phi: Y_{1} \times I \rightarrow \mathbb{R}^{n+1} \times I$, where $Y_{1}$ is an $n$-disk.

Suppose we have a fixed value of $n$; we now describe the collection $\mathcal{M}^{n}$ of standard local moves needed for the proof of Proposition 2.9; there is one such local move for each singularity type as described above.

The standard local moves for the other types of singularities now proceed using the constructions described in the previous two cases, modified to take into account that we need to consider more than one or two disks, that we may have mixed types, and that these disks have to be in general position. For example, for a singularity of type $S(c, 3, p)$, we would begin by defining $f_{1}, f_{2}$ and $f_{3}$ by

$$
\begin{aligned}
& f_{1}\left(\left(t_{1}, \ldots, t_{n}\right), s\right)=\left(\left(t_{1}, \ldots, t_{n}, s, 1\right), \mu_{p}^{n-1}\left(t_{2}, \ldots, t_{n}\right)-s-t_{1}\right), \\
& f_{2}\left(\left(t_{1}, \ldots, t_{n}\right), s\right)=\left(\left(t_{1}, \ldots, t_{n}, s, 0\right), \mu_{p}^{n-1}\left(t_{2}, \ldots, t_{n}\right)+s-t_{1}\right), \\
& f_{3}\left(\left(t_{1}, \ldots, t_{n}\right), s\right)=\left(\left(t_{1}, \ldots, t_{n}, s, 2\right), \mu_{p}^{n-1}\left(t_{2}, \ldots, t_{n}\right)+s+t_{1}\right) .
\end{aligned}
$$

As before, by examining the last coordinate of the $\mathbb{R}^{n+2}$ factor, we see that the images of the $f_{i}$ are disjoint. The images of $P \circ f_{1}$ and $P \circ f_{2}$ intersect in an $n$-disk which is the image of those points of the form $\left(\left(t_{1}, \ldots, t_{n}\right), 0\right)$. Also, $P \circ f_{1}$ and $P \circ f_{3}$ intersect in an $n$-disk which is the image of points of the form $\left(\left(t_{1}, \ldots, t_{n}\right),-t\right)$. In addition, $P \circ f_{2}$ and $P \circ f_{3}$ intersect in an $n$-disk which is the image of points of the form $\left(\left(0, \ldots, t_{n}\right), s\right)$. The intersection of all three is an $(n-1)$-disk which is the image of points of the form $\left(\left(0, t_{2}, \ldots, t_{n}\right), 0\right)$, and on that set the $\mathbb{R}^{1}$ coordinate is given by $\mu_{p}^{n-1}$.

7. Local models for knot moves. The following lemma is a straightforward generalization of "Morse's Lemma" (see, for example, [HR2] for 
statement of the latter). In fact, if $q=0$ Proposition 7.1 is exactly the Morse Lemma.

Proposition 7.1. View $\mathbb{R}^{m}$ as $\mathbb{R}^{q} \times \mathbb{R}^{m-q}$ and use coordinates $\left(x_{1}, \ldots, x_{m}\right)$ for points of $\mathbb{R}^{m}$. Suppose $f: \mathbb{R}^{m} \rightarrow \mathbb{R}^{1}$ is a smooth function such that $f(0)=0, f \mid \mathbb{R}^{q} \times\{0\}$ is a Morse function with a singular critical point of index $p$ at 0 , and $f \mid \mathbb{R}^{q} \times\{0\}$ is nonsingular. Then there is an open set $U$ in $\mathbb{R}^{m}$ with $0 \in U$ and an embedding $\phi: U \rightarrow \mathbb{R}^{m}$ such that

$$
\phi \circ f\left(x_{1}, \ldots, x_{m}\right)=\sum_{i=1}^{p}-x_{i}^{2}+\sum_{i=p+1}^{q} x_{i}^{2}+\sum_{i=q+1}^{m}-\varepsilon_{i} x_{i},
$$

where $\varepsilon_{i}= \pm 1$.

REMARK 7.2. The choice of $\varepsilon_{i}$ to be +1 or -1 can be any desired one, since if we are given one such $\phi$ as in the lemma we may obtain another coordinate chart with the direction of the $i$ th coordinate reversed, and this has the effect of changing the sign of $\varepsilon_{i}$ (if $q+1 \leq i \leq m$ ).

We are now ready to prove Proposition 2.9. Using Proposition 5.4, we replace the given isotopy by an isotopy $F: M^{n} \times I \rightarrow \mathbb{R}^{n+2} \times I$ arranged for moves. Let $x_{1}^{*}, \ldots, x_{r}^{*}$ be the elementary singularities, where $p\left(x_{i}^{*}\right)<p\left(x_{j}^{*}\right)$ if $i<j$. Since $F$ is in general position with respect to projection, about each $x_{i}^{*}$ we may find a neighborhood $B_{i} \times I_{i}$, where $B_{i}$ is an $(n+1)$-ball in $\mathbb{R}^{n+1}$ and $I_{i}=\left[a_{i}, b_{i}\right]$, such that $\left(\pi^{\prime} \circ F\right)^{-1}\left(B_{i} \times I_{i}\right)=Y_{i} \times I_{i}$, where $Y_{i}$ is a disjoint union of $n$-disks, $Y_{i}=\bigcup_{k} Y^{(k)}$. We show below that by perhaps choosing smaller balls $B_{i} \times I_{i}$ and $\left\{Y_{i}^{(k)}\right\}$ (and reparameterizing $I_{i}$ to $I$ ) $F \mid Y_{i} \times I$ projects equivalently to the standard local move of $\mathcal{M}^{n}$ corresponding to the singularity type of $x_{i}^{*}$. It is this isotopy which is our $\mu_{i}$. Since $p\left(x_{i}^{*}\right)$ is the only critical value in $I_{i}$, outside $Y_{i}$ the isotopy will project without changes. Thus $\mu_{i}$ is a move (with respect to $c_{i}=B_{i} \times \mathbb{R}^{1}$ ). Furthermore if we let $I_{0}^{\prime}=[0, a], I_{i}^{\prime}=\left[a_{i}, b_{i+1}\right]$ and $I_{r}^{\prime}=\left[b_{r}, 1\right]$, then $F \mid M^{n} \times I_{i}^{\prime}$ projects without changes and we let $\nu_{i}$ correspond to $F$, where we reparameterize $I_{i}^{\prime}$ to $I$.

Consider the case where a given singularity $x_{i}^{*}$ is of type $S(c, 2, p)$; then we have a map $F \mid\left(Y_{1} \cup Y_{2}\right) \times I_{i}$ into $\mathbb{R}^{n+2} \times I$. We find local coordinates for $Y_{j}$ and $\mathbb{R}^{n+2}$ so that $F$ is given by coordinates as described for the corresponding standard local move. Consider the $n$-disk $Y_{1}$. We assume that the subscripts on $Y_{j}$ have been chosen so that $F\left(Y_{1} \times I_{i}\right)$ lies below $F\left(Y_{2} \times I_{i}\right)$ with respect to the projection $\pi^{\prime}$.

Let $x_{j}=\left(\pi^{\prime} \circ F\right)^{-1}\left(x_{i}^{*}\right) \cap Y_{j}$. Next we note that, by changing the isotopy $F$ slightly, we may find a neighborhood $N_{1}$ of $x_{1}$ so that if $q: \mathbb{R}^{n+2} \times I$ $\rightarrow \mathbb{R}^{n+1}$ is projection onto the first $(n+1)$-coordinates then $(q \circ F) \mid N_{1}$ is an embedding. To see this, give points of $Y_{1} \times I_{i}$ coordinates $\left(\left(t_{1}, \ldots, t_{n}\right), s\right)$ 
so that $x_{1}=\left(0, \ldots, 0, a_{s}\right)$. Since $F$ is level preserving, $x_{1}$ is a critical point of $p$, and $Y_{1}$ contains no branch points. Then $q \circ F$ embeds the $n$-dimensional subspace $X=\left(\left(t_{1}, \ldots, t_{n}\right), a_{s}\right)$. Let $\vec{u} \in \mathbb{R}^{n+1}$ be a unit vector normal to $q \circ F(X)$ at $x_{i}^{*}$. Write $\vec{u}=\left(u_{1}, \ldots, u_{n}\right)$. The only reason that $q \circ F$ would fail to be an embedding near $x_{i}$ is that the isotopy $F_{s}$ (where $F_{s}=F \mid M \times\{s\}$ ) might be (instantaneously) stationary at $\left(0_{1}, \ldots, 0_{n}\right)$ for $s=a_{s}$, or is moving in a direction which projects to the tangent plane of $(q \circ F)(X)$. This can be remedied if necessary by adding a small linear push in the direction $\vec{u}^{\prime}$ in $\mathbb{R}^{n+2}$ where $\vec{u}^{\prime}=\left(u_{1}, \ldots, u_{n+1}, 0\right)$. More specifically, we write $F \mid Y_{i} \times I_{i}$ as $F=\left(F_{1}, \ldots, F_{n+2}, F_{n+3}\right)$. Define $\varepsilon(s)$ to be a smooth function from $\left[a_{i}, b_{i}\right]$ to $\mathbb{R}^{n+2}$ with $\varepsilon\left(a_{i}\right)=\varepsilon\left(b_{i}\right)=0 ; \varepsilon(s)=\varepsilon\left(x-a_{s}\right)$ on some interval containing $a_{s}$, where $\varepsilon$ is a small positive number; $\varepsilon(s)$ is monotone otherwise. Then

$$
F^{\prime}=\left(F_{1}+\varepsilon u_{1}, \ldots, F_{n+1}+\varepsilon u_{n+1}, F_{n+2}, F_{n+3}\right)
$$

is an isotopy equivalent to $F$ which still satisfies the previous hypotheses, and $q \circ F^{\prime}$ is an embedding in a neighborhood $N_{1}$ of $x_{1}$. We may now use this diffeomorphism to give coordinates to a small ball $B_{i}$ about $x_{2}^{*}$ so that the embedding $F \mid Y_{1} \times I_{i}$ is given by

$$
F\left(\left(t_{1}, \ldots, t_{n}\right), s\right)=\left(\left(t_{1}, \ldots, t_{n}, s, h\left(t_{1}, \ldots, t_{n}, s\right)\right), f\left(t_{1}, \ldots, t_{n}, s\right)\right),
$$

where $h$ is the height function associated with the projection $\pi^{\prime}$ and $F$ satisfies the conditions of Lemma 7.1. Without loss of generality, we may assume that the height of the point $F\left(x_{1}\right)$ is zero and the height function is the constant zero. By Proposition 7.1, in a neighborhood of $x_{1}$ we may find coordinates so that

$$
f\left(\left(t_{1}, \ldots, t_{n}\right), s\right)=\mu_{p}^{n}\left(t_{1}, \ldots, t_{n}\right)-\left(s-a_{s}\right)
$$

and by reparameterization in the last coordinate we see that the disk $Y_{1} \times I$ is embedded equivalently to the corresponding disk in the standard local model. This is not quite enough - we need the disk $Y_{2} \times I$ to be mapped just like the other disk in the standard model. We follow basically the same procedure. Our problem concerning $q \circ F$ being a local embedding would be a problem in at most one of our disks, $Y_{1} \times I$ or $Y_{2} \times I$, since in the projection they are transverse. Since $F\left(Y_{1} \times I\right)$ lies above $F\left(Y_{2} \times I\right)$, we may assume $F\left(x_{2}\right)$ has height 1 and this allows us to get, in a small neighborhood, coordinates so that the height is 1 there. Finally, an examination of the transversality of $\left(Y_{1} \times I_{i}\right)^{*}$ and $\left(Y_{2} \times I_{i}\right)^{*}$ shows that if we choose coordinates to express $F \mid Y_{1} \times I_{i}$ as above then we may use the same coordinates for $\mathbb{R}^{n+2} \times I$ and appropriate ones for $Y_{2} \times I$ so that on $Y_{2} \times I, F$ is given by

$$
F\left(\left(t_{1}, \ldots, t_{n}\right), s\right)=\left(\left(t_{1}, \ldots, t_{n}, s, 1\right), \mu_{p}^{n}\left(t_{1}, \ldots, t_{n}\right)+\left(s-b_{s}\right)\right),
$$

where $x_{2}$ has coordinates $\left(0, \ldots, 0, b_{s}\right)$. 
Next we consider the case where $x_{i}^{*}$ is a singularity of type $S(b, 1, p, q)$. Let $x=\left(\pi^{\prime} \circ F\right)^{-1} x_{i}^{*}$. Then we have a map $F: Y_{1} \times I_{i} \rightarrow \mathbb{R}^{n+2} \times I_{i}$, where $Y_{i}$ is an $n$-disk with coordinates $\left(\left(t_{1}, \ldots, t_{n-1}, x\right), s\right)$ chosen so that $x$ has coordinates $\left((0, \ldots, 0), a_{s}\right)$. Let $X$ denote the hyperplane $\left(\left(t_{1}, \ldots, t_{n}\right), a_{s}\right)$ and consider the map $q \circ F \mid X$ into $\mathbb{R}^{n+1}$. Since the derivative of $F$ in the $I$ direction is nonzero (since $F$ is an isotopy) it follows that $q \circ F$ has corank zero or one, and using $[\mathrm{MR}]$ we may find coordinates for $\mathbb{R}^{n+1}$ so that $q \circ F$ is given by

$$
\left(t_{1}, \ldots, t_{n-1}, x\right) \mapsto\left(t_{1}, \ldots, t_{n-1}, t_{1} x, x^{2}\right) .
$$

Thus we may choose coordinates so that, locally, $F: Y_{i} \times I_{i} \rightarrow \mathbb{R}^{n+2} \times I$ is given by

$$
\left(\left(t_{1}, \ldots, t_{n-1}, x\right), s\right) \mapsto\left(\left(t_{1}, \ldots, t_{n-1}, t_{1} x, x^{2}, h\right), f\right) .
$$

Now $h$ is the height function of the projection $\pi^{\prime}$. Any height function $h^{\prime}$ of this projection yields equivalent embedding as long as for each double point $x^{*}$ the height of the over point corresponding to $x^{*}$ is greater than the height of the corresponding under point. Thus by orienting our $x$-coordinate in the appropriate direction, we may assume that $h\left(\left(t_{1}, \ldots, t_{n-1}, x\right), s\right)=x$ (recall the double point set corresponds to $t_{1}=0$ ). By a previous remark, we note that $x^{*}$ is a critical point of the double point set as well as for the branch set so that the restriction of the map $F$ to the subset corresponding to $t_{1}=0, s=a_{n}$ is a Morse function of $n-l$ variables with index $p+\varepsilon$, where $\varepsilon= \pm 1$. Thus using Proposition 7.1 we may find coordinates so that

$$
\begin{aligned}
& f\left(\left(t_{1}, \ldots, t_{n-1}, x\right), s\right) \\
& \quad=\left(\left(t_{1}, \ldots, t_{n-1}, t_{1} x, x^{2}, s-a_{s}\right), \mu_{p}^{n-1}\left(t_{1}, \ldots, t_{n-1}\right)+\varepsilon x^{2}+\left(s-a_{s}\right)\right) .
\end{aligned}
$$

Thus we have found coordinates as in the standard local model for a singularity of this type.

The cases of singularities of other types clearly follow by straightforward use of the techniques in the above two cases. We omit these details, thus concluding our sketch of the proof of Proposition 2.9.

There is a further device for reducing the number of knot moves to consider.

Definition 7.3. If $F: Y \times I \rightarrow \mathbb{R}^{n+2} \times I$ represents a knot move, then the inverse of $F$ is the isotopy $F^{\prime}(y, t)=F(y, 1-t)$. If $F$ corresponds to a singularity of type $S(c, 2, p)$ then $F^{\prime}$ corresponds to a singularity of type $S(c, 2, n-p)$. If $F$ corresponds to a singularity of type $S(b, 1, p, q)$ then $F^{\prime}$ corresponds to a singularity of type $S(b, 1,(n-1)-p, 1-q)$, etc. We say that the knot moves of $F$ and $F^{\prime}$ are of the same type. 
Thus the number of types of moves is roughly half the number of elements of $\mathcal{M}^{n}$. With this definition, the knot moves in low dimensions corresponding to Proposition 2.9 are listed in Propositions 8.1 and 8.2. A more detailed exposition of the case of knotted surfaces in $\mathbb{R}^{4}$ as well as discussion of knotted three-dimensional manifolds in $\mathbb{R}^{5}$ may be found in [RS2].

8. Knotted circles in $\mathbb{R}^{3}$ and knotted surfaces in $\mathbb{R}^{4}$. We now wish to briefly consider the knot moves in dimensions $n=1,2$. We first note that Proposition 2.9 gives us the classical set of Reidemeister moves.

Proposition 8.1. If $F: M^{1} \times I \rightarrow \mathbb{R}^{3} \times I$ is an isotopy of a closed one-dimensional manifold (i.e., a classical knot or link) and if $F \mid M \times\{0\}$ and $F \mid M \times\{1\}$ are in general position with respect to projection, then $F$ is equivalent to an isotopy by elementary moves, where these moves are of three types which we list below by the corresponding singularities:

1. A local maximum or a local minimum of the crossing set $D^{*}$.

2. A point of $B^{*}$, and either a local maximum or local minimum of $D^{*}$.

3. A point of the crossing set of $D^{*}$.

Here case 2 corresponds to $\Omega_{1}$, case 1 corresponds to $\Omega_{2}$, and case 3 corresponds to $\Omega_{3}$ of Reidemeister. In these dimensions we may draw pictures of images of the projections of the corresponding knot moves, that is, images of $f: Y \times I \rightarrow \mathbb{R}^{2} \times I$ for the various standard models (see Figure 4; here the vertical direction is the $I$ coordinate; slicing horizontally near the critical points, labeled $x^{*}$, gives the classical knot moves).

Proposition 8.2. If $F: M^{2} \times I \rightarrow \mathbb{R}^{4} \times I$ is an isotopy of a closed twodimensional manifold and if $F \mid M^{2} \times\{0\}$ and $F \mid M^{2} \times\{1\}$ are in general position with respect to projection, then $F$ is equivalent to an isotopy by elementary moves, where these moves are of seven types which we list below by the corresponding singularities:

1. Local maximum or local minimum of the crossing set $D^{*}$.

2. Saddle point of $D^{*}$.

3. Local maximum or local minimum of the branch set $B^{*}$ which is a local maximum or local minimum (respectively) of $D^{*}$ in direction transverse to $B^{*}$.

4. Local maximum or local minimum of $B^{*}$ which is a local minimum or local maximum (respectively) of $D^{*}$ in direction transverse to $B^{*}$.

5. Local maximum or local minimum of the crossing set of $D^{*}$.

6. A point where $B^{*}$ meets the crossing set of $D^{*}$.

7. A triple point of $D^{*}$. 


\section{References}

[GR] C. Gordon, Some aspects of classical knot theory, in: Knot Theory, Lecture Notes in Math. 685, Springer, 1978, 347-380.

[HR] M. Hirsch, Differential Topology, Grad. Texts in Math. 33, Springer, 1976.

[ML] J. Milnor, Morse Theory, Ann. of Math. Stud. 51, Princeton Univ. Press, 1963.

[MR] B. Morin, Formes canoniques des singularités d'une application différentiable, C. R. Acad. Sci. Paris 260 (1965), 5662-5665.

[NB] G. Naber, Topological Methods in Euclidian Spaces, Cambridge Univ. Press, 1980.

[RD] K. Reidemeister, Knotentheorie, Springer, 1932; reprint, Springer, 1974.

[RS1] D. Roseman, Projections of codimension two embeddings, in: Knots in Hellas '98, Ser. Knots Everything 24, World Sci., 2000, 380-410.

[RS2] - Reidemeister-type moves for surfaces in four-dimensional space, in: Knot Theory, V. F. R. Jones et al. (eds.), Banach Center Publ. 42, Inst. Math. Polish Acad. Sci., 1998, 347-380.

The University of Iowa

Iowa City, IA 52242, U.S.A.

E-mail: roseman@math.uiowa.edu

Received 7 May 2004;

in revised form 30 June 2004 